\title{
De-implementation of inappropriately tight control (of hypoglycemia) for health: protocol with an example of a research grant application
}

\author{
David C Aron ${ }^{1 *}$, Julie Lowery ${ }^{2}$, Chin-lin Tseng ${ }^{3}$, Paul Conlin ${ }^{4}$ and Leila Kahwati ${ }^{5}$
}

\begin{abstract}
Background: Implementation of practice change is difficult and large scale implementation is particularly difficult. Among the challenges facing the healthcare system in general and healthcare organizations is the overuse of low value care. Improving medication safety also constitutes an attempt to reduce low value or potentially harmful care. Critical issues of overuse of low value practices and medication safety intersect in overtreatment of diabetes. Specifically, (over)intensive glycemic control increases hypoglycemia risk and morbidity without providing meaningful benefit. Our work indicates that among patients with diabetes who are at high risk for hypoglycemia, potential overtreatment is common. The Choosing Wisely Initiative to reduce low value care led by the American Board of Internal Medicine Foundation recommends not to treat most persons over 65 years of age with medications to reduce the $\mathrm{A} 1 \mathrm{c}<7.5 \%$. For most physicians this involves a change in practice. We will study the implementation of the Veterans Health Administration's Choosing Wisely Initiative (which includes hypoglycemic safety as a targeted condition) with three specific aims: (1) to assess the overall impact, both intended and unintended, of the Choosing Wisely Initiative to reduce overtreatment of diabetes in especially vulnerable populations; (2) to assess the impact of commitment to quality, teaching intensity, and safety culture on likelihood of overtreatment; and (3) to identify configurations of the implementation strategy, provider characteristics and organizational level factors that are associated with successful reduction of overtreatment rates by comparing high and low performers. Because focus on this initiative could have the unintended consequence of paying less attention to poor glycemic control (A1c>9\%), we will also assess undertreatment.
\end{abstract}

Methods/Design: We will take advantage of a natural experiment and use a Type III Hybrid Design that focuses on study of implementation while at the same time observing and gathering information on clinical interventions and outcomes. This mixed methods study will use longitudinal data and qualitative methods including Qualitative Comparative Analyses.

Discussion: Our multi-paradigm approach to examining potential mechanisms to explain the variation in reduction of rates of overtreatment will contribute to a better understanding of implementation of national dissemination projects and multi-component interventions in complex systems.

\footnotetext{
* Correspondence: david.aron@va.gov

${ }^{1}$ Interprofessional Implementation Research, Evaluation and Clinical Center, Louis Stokes Cleveland Department of Veterans Affairs Medical Center (EUL Room 5M677), 10701 East Boulevard, Cleveland, OH 44106, USA

Full list of author information is available at the end of the article
} 
Table 1 Ten key ingredients for implementation research proposals (modified from Proctor EK et al. [1])

Ten key ingredients
Evidence of a gap in quality

\section{How ingredients are operationalized}

In addition to support from the literature, research team members were able to cite their own work about potential overtreatment of diabetes. Another gap is in knowledge about how to de-implement well-established practices.

Evidence-based treatment to be implemented

Conceptual model and theoretical justification

Stakeholder priorities, engagement in change

Setting's readiness to adopt new services/ treatments/programs

Implementation strategy/process

Team experience with the setting, treatment, implementation process

Feasibility of proposed research design and methods

Measurement and analysis section

Policy/funding environment; leverage or support for sustaining change

There are many guides to developing research proposals, and ingredients for implementation research grant proposals have been suggested by Proctor et al. [1]. However, among the challenges facing a new investigator in trying to get research funding is the relative paucity of model grant applications $[2,3]$. This is particularly true when the field being entered is a newly developing discipline; implementation science is such a discipline and it is still evolving [4]. Although the publication of protocols from such grant applications has become more common, the actual grant application and its iterations have not. Our goal is not only to provide an example of an implementation research grant application, but also to illustrate this process further by making available the different iterations and the critiques as well. In so doing, we take the process one step further by illustrating how the research team revised the application in response to the critiques. Each funding agency has its own application procedures. These procedures may differ in the details, but are similar. Some require 'letters of intent' or 'concept papers' while others do not. The revised application is in Additional file 1. We have included in the six other additional files labeled: Additional files 2, 3, 4, 5, 6 and 7.' This process lasted from May 2013 to March 2014. Of note, in the middle of the process, the funding agency changed its requirements between initial and revised submissions in terms of the length of the narrative, reducing it from 25 to 15 pages.

Proctor et al. identified ten ingredients of a successful implementation research grant proposal [1]. These ingredients are listed in Table 1. All ten ingredients were included in our application to varying degrees. No claims are made that this is the optimal proposal that could be written on the subject, merely that it suffices, i.e., it was good enough to have been funded successfully [5], and they are highlighted in Table 1. It is the hope of the authors that making this material available, with all its imperfections, will foster development of this crucially important discipline. It should also be stated that the process of submission and review resulted in what we believe is a much improved proposal.

\section{Additional files}

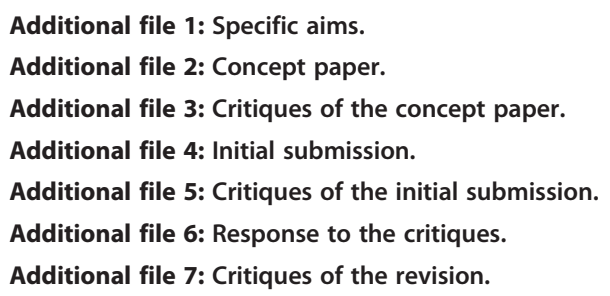




\section{Competing interest}

The authors declare that they have no competing interests. The views expressed are those of the author and do not represent the views of the Dept. of Veterans Affairs or any other agency.

\section{Authors' contributions}

DA drafted the introductory article. For the additional files constituting the grant application, DA (Principal Investigator) drafted the first version. JL

(co-Investigator) drafted the section on qualitative methods. LK (co-Investigator) drafted the section on qualitative comparative analysis. C-LT (co-Investigator) drafted the statistical methods section. PC contributed to the writing. Multiple drafts of the grant application were reviewed and critically by all authors. The authors of the critiques were anonymous. All authors read and approved the final manuscript.

\section{Author details}

'Interprofessional Implementation Research, Evaluation and Clinical Center, Louis Stokes Cleveland Department of Veterans Affairs Medical Center (EUL Room 5M677), 10701 East Boulevard, Cleveland, OH 44106, USA. VA Health Services Research \& Development Quality Enhancement Research Initiative-Diabetes, VA Ann Arbor Health Care System, 2215 Fuller Road, Ann Arbor, MI, USA. ${ }^{3}$ Research Service, Department of Veterans Affairs, New Jersey Health Care System, 385 Tremont Ave., East Orange, NJ, USA. ${ }^{4}$ Medical Service, VA Boston Healthcare System, Jamaica Plain Campus 150 South Huntington Avenue, Jamaica Plain, MA, USA. ${ }^{5}$ TTI International, Research Triangle Park, NC, USA.

Received: 3 April 2014 Accepted: 8 May 2014

Published: 19 May 2014

\section{References}

1. Proctor EK, Powell BJ, Baumann AA, Hamilton AM, Santens RL: Writing implementation research grant proposals: ten key ingredients. Implement Sci 2012, 7:96.

2. Naylor MD: Special feature: an example of a research grant application: comprehensive discharge planning for the elderly. Res Nurs Health 1990, 13:327-347.

3. Annotated R01 Grant Application - NIAID: Bethesda MD, USA: National Institute of Allergy and Infectious Diseases, National Institutes of Health. http://www.niaid.nih.gov/ncn/grants/app/default.htm. 8-21-2013. Accessed 3-24-2014.

4. Bammer G: Integration and implementation sciences: building a new specialization. Ecol Soc 2005, 10(2):6.

5. Simon HA: The Sciences of the Artificial. 3rd edition. Cambridge, MA: MIT Press; 1996.

doi:10.1186/1748-5908-9-58

Cite this article as: Aron et al:: De-implementation of inappropriately tight control (of hypoglycemia) for health: protocol with an example of a research grant application. Implementation Science 2014 9:58.

\section{Submit your next manuscript to BioMed Central and take full advantage of:}

- Convenient online submission

- Thorough peer review

- No space constraints or color figure charges

- Immediate publication on acceptance

- Inclusion in PubMed, CAS, Scopus and Google Scholar

- Research which is freely available for redistribution 